\title{
Associated Myocarditis: A Predictive Factor for Response?
}

\author{
Walid Shalata $^{\mathrm{a}}$ Nir Peled $^{\mathrm{a}} \quad$ Itzhak Gabizon $^{\mathrm{b}}$ Omar Abu Saleh ${ }^{\mathrm{c}}$ \\ Waleed Kian $^{a} \quad$ Alexander Yakobson $^{\mathrm{a}}$ \\ aThe Legacy Heritage Oncology Center and Dr. Larry Norton Institute, Soroka Medical \\ Center and Ben-Gurion University, Beer Sheva, Israel; ${ }^{b}$ Department of Cardiology, Soroka \\ University Medical Center, Faculty of Health Sciences, Ben-Gurion University of the Negev, \\ Beer Sheva, Israel; 'Department of Dermatology and Venereology, The Emek Medical \\ Centre, Afula, Israel
}

\section{Keywords}

Immune checkpoint inhibitors · Myocarditis · Melanoma · Pembrolizumab · Nivolumab · Ipilimumab

\section{Abstract}

In the present case report, we aimed to describe 2 cases of myocarditis occurring as serious adverse effects of immune checkpoint inhibitors (ICls) administered as treatment for metastatic melanoma. We describe 2 female patients: an 81-year-old treated with pembrolizumab and a 55-year-old treated with a combination of nivolumab and ipilimumab. Both patients underwent resection of metastases; while under treatment, both developed myocarditis, most probably as a toxicity from pembrolizumab and nivolumab plus Ipilimumab, respectively. While they achieved complete response, the occurrence of myocarditis as a toxicity of $\mathrm{ICls}$ may have been a predictive sign that the immune system was sufficiently activated by the checkpoint inhibitor therapy to induce complete remission.

\section{Introduction}

Cutaneous melanoma, a malignancy whose origin is the pigment-producing cell in the skin, the melanocyte [1], accounts for $1 \%$ of all skin cancers [2]. Most melanoma cells continue to synthesize melanin, so melanoma tumors are usually brown or black. However, some melanomas do not make melanin and can appear pink, tan, or even white. 
Melanomas can develop anywhere on the skin, but they are more likely to start on the trunk (chest and back) in men and on the legs in women. The neck and face are other common sites [1]. An estimated 9,320 people die of melanoma annually in the USA, 5,990 men and 3,330 women. From 2006 to 2015, the death rate for melanoma declined by $1 \%$ per year in adults 50 years of age and older and by $2.6 \%$ per year in those younger than 50 years [3].

Metastatic melanoma can show widespread organ involvement: the lymph nodes are involved in $70-75 \%$ of cases of metastatic melanoma, areas of the skin, fat, and muscle in $65-70 \%$, the lung in $70-87 \%$, liver and gallbladder in $54-77 \%$, the brain in $36-54 \%$, bone in $23-49 \%$, the gastrointestinal tract in $26-58 \%$, the heart in $40-45 \%$, the pancreas in $38-53 \%$, the adrenal glands in $36-54 \%$, the kidneys in $35-48 \%$, the spleen in $30 \%$, and the thyroid in $25-39 \%$ [4].

Over the past decade, there has been a revolution in the systemic treatment of metastatic melanoma, and patients now have more options for more effective treatment, such as targeted therapy and immunotherapy. In addition, molecular tests have been developed to select the most appropriate therapy for the individual patient.

Immune checkpoint inhibitors (ICIs) have been approved by regulatory authorities around the world for an extended range of malignancies. Along with substantially improved treatment results, including progression-free survival, overall survival, and clinical benefit, the ICIs also have led to immune-related adverse effects that in a minority of cases are lifethreatening.

Myocarditis (an inflammation of the heart muscle [myocardium]) is an uncommon but potentially fatal complication of ICI therapy in which the patient may develop heart failure, QT prolongation, arrhythmias, left ventricular (LV) hypertrophy, myocardial ischemia, and infarction. However, generally, the toxicity resolves after treatment discontinuation.

The incidence of ICI-associated myocarditis is low and has been reported to range from 0.06 to $1 \%$ in patients receiving an ICI [5]. The prevalence of myocarditis as reported in a recent study was $1.14 \%$ with a median time of onset of 34 days after starting ICI (interquartile range 21-75) [6]. Myocarditis may be difficult to diagnose, so it is possible that the actual incidence is higher [5].

\section{Case 1}

A 78-year-old woman underwent excision of an ulcerated bleeding skin lesion in the left thigh area in 2015. She had noted a black lesion on her thigh since 2010. She had been treated for hypertension and hypothyroidism. She had no family history of cancer and did not smoke.

The excisional biopsy showed ulcerated nodular malignant melanoma Clark Level 4. Subsequently, on November 29, 2015, she underwent wide excision of the area of the biopsy with sentinel lymph node biopsy from the left inguinal area. The pathological examination showed a few isolated metastatic melanoma cells in one lymph node. No tumor was seen in the area of the surgical scar of the previous biopsy.

In December 2015, she was referred to the Oncology Department for evaluation and treatment. Combined positron emission tomography-computed tomography (PET-CT) test showed multiple pulmonary nodules with the largest diameters up to $5 \mathrm{~mm}$ in the right upper lobe and right lower lobe as well as "ground glass" shading at the top of the lung. The findings were not felt to represent malignant disease and no other abnormalities were found. The patient was diagnosed and staged as malignant nodular-type ulcerated melanoma of the left thigh, T3b N1mic M0 (stage 3b).

PET-CT in February 2018 showed metastases in the lungs and thoracic spine (vertebrae D10-D11) and lymphadenopathy in the left inguinal area consistent with the diagnosis of metastatic malignant melanoma. We initiated systemic therapy with pembrolizumab $150 \mathrm{mg}$ intravenously every 3 weeks as immunotherapy treatment on February 28, 2018. She was 
Shalata et al.: Immune Checkpoint Inhibitor and Myocarditis
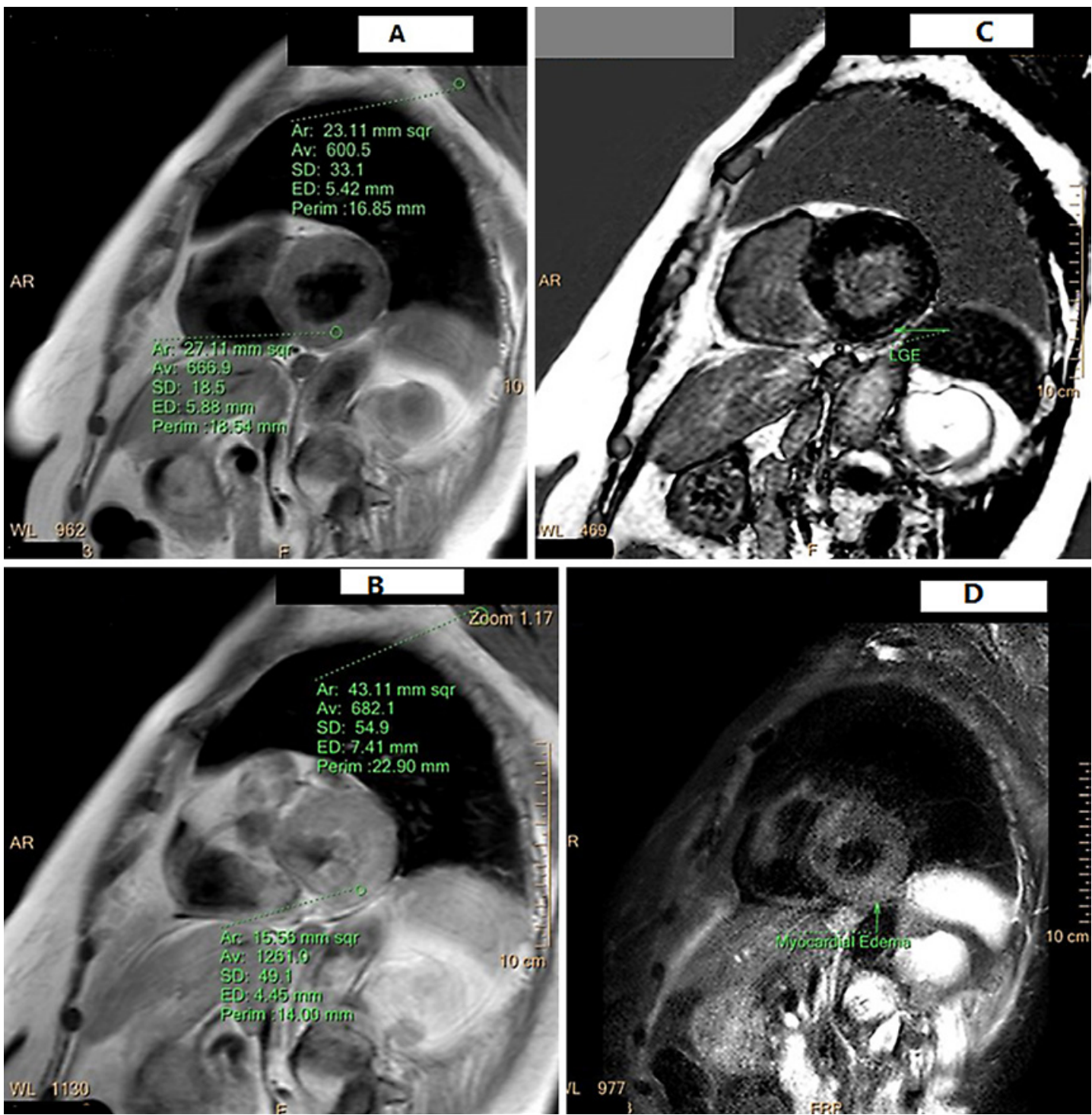

Fig. 1. Myocarditis in patient 1. A, B Difference before (A) and after (B) late gadolinium-enhanced cardiac MRI of the patient, which is a sign of myocarditis (arrow). C, D Late gadolinium enhancement in a pattern compatible with myocarditis (injection of gadolinium showing myocardial hyperemia with an area of more pronounced uptake [arrow]).

given palliative radiotherapy to vertebrae D9-L1 (20 Gy, 5 fractions). Orthopedic consultation did not recommend invasive palliative treatment for the spine metastases.

On March 26, 2018, she received the second cycle of pembrolizumab immunotherapy treatment. On April 14, 2018, she was admitted to the Department of Internal Medicine due to chest pain. After 5 days, she was transferred to the intensive care unit due to suspicion of non-ST-segment elevation myocardial infarction with troponin level $490 \mathrm{ng} / \mathrm{L}$ (normal 0.0-14.0), which was increasing. Cardiac catheterization demonstrated nonobstructive coronary disease and a hyperdynamic LV function. An echocardiogram was done the same day showing normal LV systolic function with upper septal hypertrophy and elevated LV filling pressure. 
Fig. 2. Normal coronary arteries in an angiogram of patient 1 .

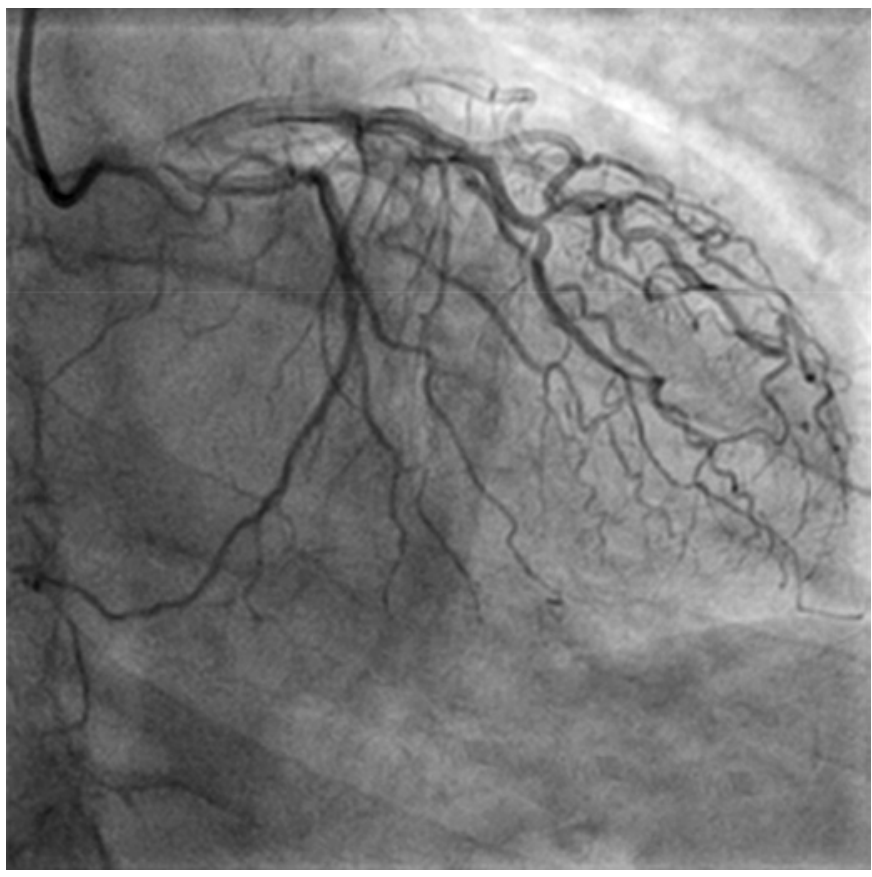

On April 18, 2018, a cardiac magnetic resonance imaging (MRI) was preformed that demonstrated (Fig. 1) normal LV systolic function with septal hypertrophy. The early gadolinium enhancement (Fig. 1A) ratio was $>4$ with late gadolinium enhancement (Fig. 1B), in a pattern compatible with myocarditis (Fig. 1C, D).

Corticosteroid therapy was initiated with prednisone $2 \mathrm{mg}$ per kilogram body weight for 1 week, and because of the suspicion that the myocarditis was caused by pembrolizumab, prednisone was continued for 2 weeks at a dose of $1 \mathrm{mg} / \mathrm{kg}$.

Troponin level decreased from 1,103 ng/L to $300 \mathrm{ng} / \mathrm{L}$. Virology testing was performed (hepatitis serology, Q-FEVER phases). DNA PCR for EBV and CMV was negative, HIV was negative, and Brucella abortus with Rose Bengal test was not detected.

Two weeks later, she had an echocardiogram that showed normal LV systolic function. An angiogram of the coronary arteries showed normal coronary arteries (Fig. 2).

A multidisciplinary conference including a cardiologist, oncologist, and infectious disease specialist came to the conclusion that the clinical presentation of the patient was consistent with myocarditis as a toxicity of immunotherapy. Pembrolizumab was discontinued.

On May 25, 2018, troponin level was $100 \mathrm{ng} / \mathrm{L}$, and complete blood count and thyroid function were within normal limits. On June 28, 2018, laboratory results showed an elevated troponin level of $131 \mathrm{ng} / \mathrm{L}$, with no other abnormalities. On July 16, 2018, CT of the chest, abdomen, and pelvis showed no vertebral metastasis and no other evidence of metastatic disease.

Between November 8, 2018, and November 12, 2018, she was hospitalized in the Department of Internal Medicine due to high fever $\left(39.5^{\circ} \mathrm{C}\right)$ with leukopenia $\left(2.24 \times 10^{3} / \mu \mathrm{L}\right.$, normal 4.8-10.8) and neutropenia $\left(1.4 \times 10^{3} / \mu \mathrm{L}\right.$, normal $\left.1.9-8\right)$.

CT of the total body showed no evidence of the origin of fever with no pulmonary infiltrates or metastases. Urine culture was positive for Klebsiella pneumoniae, so she was treated with antibiotics (carbapenem) and discharged home.

From November 25, 2018, to December 3, 2018, while not under active oncology treatment, she was re-admitted to the Internal Medicine Department due to repeated 
Fig. 3. Normal coronary arteries in an angiogram of patient 2 .

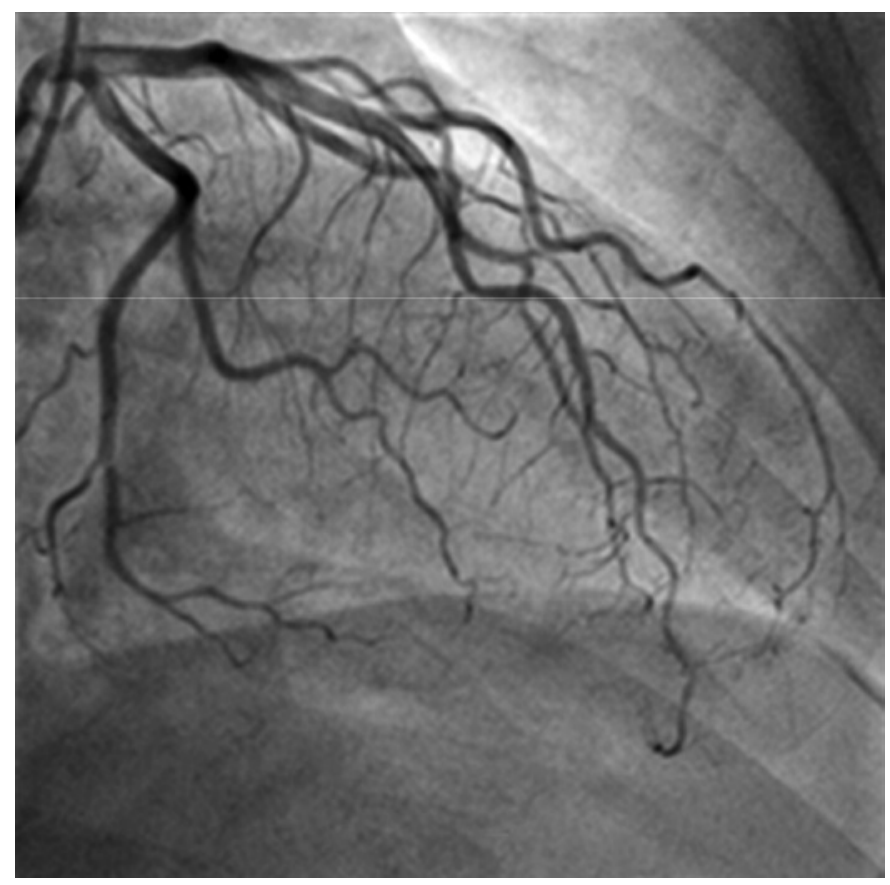

episodes of fever $\left(39.0^{\circ} \mathrm{C}\right)$. Blood and urine cultures were negative, as was a panel or serology for viruses (same panel as noted above on April 14, 2018). Echocardiogram showed no abnormalities. On December 4, 2018, CT-PET without injection of contrast material showed no evidence of any infectious process and continued absence of any signs of malignancy.

\section{Case 2}

A 55-year-old woman presented in November 2017 with a nevus $14 \mathrm{~cm}$ in diameter on the skin of her upper back. The dark blue nevus had been present since birth but recently had become larger and darker in color.

She had received treatment for hypertension and type 2 diabetes mellitus. Past medical history included total thyroidectomy in 2016 for papillary carcinoma of the thyroid and excision of a benign endometrial polyp in 2004 . She had no family history of cancer and was not a smoker.

On December 19, 2017, biopsy of the nevus showed metastatic malignant melanoma. She then underwent a total body PET-CT that showed hypermetabolic absorption in the vertebral bodies and soft tissue at levels D5, D7, and D9 (the area of the nevus) with high absorption, consistent with malignant disease.

We initiated systemic immunotherapy with nivolumab $200 \mathrm{mg}$ intravenously every 2 weeks. After the fourth cycle of treatment, she developed itching and mild skin rash. One week later, she was admitted to the Department of Internal Medicine because of headache, weakness, and increase in liver enzymes with GOT (AST) 53 U/L (normal 0-31) and GPT (ALT) 56 U/L (normal 0-34). Further evaluation included chest radiography with no evidence of pathological findings; abdominal ultrasound with no evidence of pathological findings; and CT of the abdomen with no evidence of intra-abdominal metastatic findings.

Complete blood count and chemistries were normal except for the liver enzymes previously noted as elevated, now decreasing. Hepatitis serology was negative. She was discharged home after 10 days. 
One week later, repeat PET-CT test was performed, which showed stable disease. At that point, we elected to add Ipilimumab $75 \mathrm{mg}$ and administer it together with the nivolumab 200 mg every 3 weeks.

In May 2018, she received the second cycle of nivolumab plus ipilimumab. Two weeks later, she was admitted to the Department of Internal Medicine with chest pain, fever $\left(38.7^{\circ} \mathrm{C}\right)$, and dyspnea. Electrocardiography showed a normal sinus rhythm. Echocardiogram showed normal LV systolic function with an ejection fraction of $65 \%$ and signs of impaired LV relaxation with normal LV filling pressure. Right ventricular function was preserved. There was mild mitral regurgitation and no pulmonary hypertension. Angiogram of the coronary arteries showed normal coronary arteries (Fig. 3).

Ventilation/perfusion lung scan was normal; complete blood count and blood chemistries were normal; and troponin level was high, initially $326 \mathrm{ng} / \mathrm{L}$, then decreasing to 256 $\mathrm{ng} / \mathrm{L}$. Steroid therapy was initiated (prednisone $1.5 \mathrm{mg} / \mathrm{kg}$ ). Virology testing showed the following: DNA PCR for EBV and CMV was negative, HIV was negative.

Further investigations showed the following: antinuclear antibody test was negative, anti-neutrophil cytoplasmic antibodies were negative, she had a normal immunoglobulin level, and complete blood count and blood chemistries were normal except for a mild increase in liver enzymes (GOT [AST] $80 \mathrm{U} / \mathrm{L}, \mathrm{GPT}$ [ALT] $73 \mathrm{U} / \mathrm{L}$ ) and elevated lactate dehydrogenase (843 U/L, normal 230-480). Blood culture showed no bacterial growth.

CT scan of the spine showed no evidence of metastasis. MRI of the heart showed normal LV systolic function and normal right ventricular systolic function; there was no evidence of myocardial late gadolinium enhancement and there was mild mitral regurgitation as well as minimal pericardial effusion. There were no diagnostic signs of myocarditis, but after consultation with a cardiologist, the diagnosis of myocarditis was made on clinical grounds.

On August 2018, she underwent PET-CT which showed weak hypermetabolic absorption in soft tissue in the area of the nevus. A multidisciplinary conference including an oncologist and a plastic surgeon recommended wide excision of the nevus, which was performed on September 13, 2018. The pathology results showed metastatic melanoma with negative margins.

Two months after the surgery, she was admitted for PET-CT for follow-up, which showed no evidence of disease. Presently, she is receiving nivolumab $200 \mathrm{mg}$ every 2 weeks as adjuvant therapy with no evidence of disease recurrence and normal troponin level.

\section{Discussion and Conclusion}

We have described 2 patients each of whom had a rare serious adverse event, myocarditis, known to be caused by ICIs. Both patients went on to have a complete response of widespread metastatic disease to immune therapy. Severe cardiologic side effects with immunotherapy are uncommon but are in most cases reversible; therefore, close follow-up is very important for patients on ICI treatment. We suggest that the occurrence of a severe immunerelated toxicity, myocarditis, was predictive of a dramatic tumor response to immune-based therapy.

\section{Acknowledgement}

The authors thank David B. Geffen, MD, for his critical review of the manuscript. 
Shalata et al.: Immune Checkpoint Inhibitor and Myocarditis

\section{Statement of Ethics}

All subjects have given their written informed consent to publish their case (including publication of images).

\section{Disclosure Statement}

The authors declare no conflict of interest.

\section{Funding Sources}

The authors received no financial support for the research, authorship, and/or publication of this article.

\section{Author Contributions}

Walid Shalata: Substantial contributions to the conception or design of the work; or the acquisition, analysis, or interpretation of data for the work; drafting the work or revising it critically for important intellectual content; final approval of the version to be published; agreement to be accountable for all aspects of the work in ensuring that questions related to the accuracy or integrity of any part of the work are appropriately investigated and resolved.

Nir Peled: Substantial contributions to the conception or design of the work; or the acquisition, analysis, or interpretation of data for the work; drafting the work or revising it critically for important intellectual content; final approval of the version to be published.

Itzhak Gabizon: Substantial contributions to the conception or design of the work; or the acquisition, analysis, or interpretation of data for the work; drafting the work or revising it critically for important intellectual content; agreement to be accountable for all aspects of the work in ensuring that questions related to the accuracy or integrity of any part of the work are appropriately investigated and resolved.

Omar Abu Saleh: Substantial contributions to the conception or design of the work; or the acquisition, analysis, or interpretation of data for the work; drafting the work or revising it critically for important intellectual content; final approval of the version to be published.

Waleed Kian: Substantial contributions to the conception or design of the work; or the acquisition, analysis, or interpretation of data for the work; drafting the work or revising it critically for important intellectual content; final approval of the version to be published.

Alexander Yakobson: Final approval of the version to be published; drafting the work or revising it critically for important intellectual content; agreement to be accountable for all aspects of the work in ensuring that questions related to the accuracy or integrity of any part of the work are appropriately investigated and resolved. 


\section{References}

1 Cancer.org. What is melanoma skin cancer? [Internet]. [cited 2020 Feb 29]. Available from: https://www. cancer.org/cancer/melanoma-skin-cancer/about/what-is-melanoma.html

2 Cancer.Net. Melanoma: Statistics [Internet]. 2012 [cited 2020 Feb 29]. Available from: https://www.cancer. net/cancer-types/melanoma/statistics

3 American Cancer Society Inc. Cancer facts \& figures 2018. Atlanta, GA: American Cancer Society; 2018.

4 Verywell Health. The spread of melanoma metastasis [Internet]. [cited 2020 Feb 29]. Available from: https:// www.verywellhealth.com/where-and-why-can-melanoma-spread-3010811

5 Ganatra S, Neilan TG. Immune checkpoint inhibitor-associated myocarditis. Oncologist. 2018;23(8):879-86.

6 Mahmood SS, Fradley MG, Cohen JV, Nohria A, Reynolds KL, Heinzerling LM, et al. Myocarditis in patients treated with immune checkpoint inhibitors. J Am Coll Cardiol. 2018;71(16):1755-64. 